\title{
EL ACTO MÉDICO: ¿UNA CREACIÓN ORIGINAL? REFLEXIONES SOBRE SU ESENCIA, SURGIMIENTO Y RIESGOS A LOS QUE SE EXPONE
}

\begin{abstract}
Resumen: La medicina se constituyó como una actividad enseñable con metodología propia a partir de la escuela hipocrática. Como actividad, se relaciona con todas aquellas que buscan la producción de algo, en este caso la salud de los pacientes, considerada ésta como un bien particular de ellos. El médico entonces -como todo productor- debe traspasar una intervención existente primero en su mente a un sujeto carente de salud o en riesgo de perderla. Este trabajo busca reflexionar sobre cómo consigue el médico concebir en su intelecto una acción que, plasmada en un paciente en particular, constituya para él un efecto considerado curativo. La tesis fundamental en esta reflexión es que, además de utilizar criterios útiles para un hombre considerado universalmente, debe crear una intervención diseñada a la medida de un ser humano particular, poseedor de toda una complejidad personal.
\end{abstract}

Palabras clave: ética, ética médica, filosofía de la medicina, humanidades

\section{MEDICAL ACT: AN ORIGINAL CREATION? REFLECTIONS ON ITS ESSENCE, ORIGIN AND RISKS OF EXPOSURE}

\begin{abstract}
Medicine became a teaching activity with its own methodology since the Hippocratic School. As activity, it relates to those which seek to produce something, in this case the health of patients, considering this as a particular good for them. The physician, then -as all producer- must transfer an existing intervention first in his/her mind to a subject lacking health or in risk of losing it. This study tries to reflect on how the physician achieves to imagine in his/her reasoning an action that, performed in a particular patient, it will constitute in him/her a healing effect. The fundamental thesis in this reflection is that, besides using useful criteria considering a universal human being, he/she must create an intervention designed to suit a specific human being, who has a full personal complexity.
\end{abstract}

Key words: ethics, medical ethics, philosophy of medicine, humanities

\section{O ATO MÉDICO: UMA CRIAÇÁOO ORIGINAL? REFLEXÓES SOBRE SUA ESSÊNCIA, SURGIMENTO E RISCOS A QUE SE ESTÁ EXPOSTO}

Resumen: A medicina se constituiu como uma atividade, resultante de ensino com metodologia própria, a partir da escola hipocrática. Como atividade, se relaciona com todas aquelas que buscam a produção de algo, neste caso, a saúde dos pacientes, considerada esta como um bem particular. Desse modo, o médico -enquanto produtor- deve ultrapassar à sua visão primeira, de intervenção junto a um sujeito carente de saúde ou em vías de perdê-la. Esse trabalho buscará refletir sobre como o médico consegue conceber uma ação que, plasmada em um paciente com suas particularidades, se constitua de efeito considerado curativo. A tese fundamental desta reflexão é mais que utilizar critérios úteis, considerados universais, para o ser humano, deve-se acreditar em uma intervenção desenhada na medida de um ser humano particular, possuidor de toda uma complexidade pessoal.

Palavras- chave: ética, ética médica, filosofía da medicina, humanidades

\footnotetext{
Médico Cirujano. Magíster en Filosofía. Profesor Asociado Departamento de Obstetricia y Ginecología, Centro de Bioética, Pontificia Universidad Católica de Chile, Chile

Correspondencia: besio@med.puc.cl
} 


\section{Introducción}

Cuando hablamos de medicina, espontáneamente sabemos que nos referimos al cuidado de los enfermos. Incluso, cuando se rastrean los orígenes de esta actividad, se buscan evidencias en las antiguas tumbas de señales en los restos corporales que muestren signos de intentos de curación. Así se describen piernas fracturadas que fueron alineadas, reducciones de luxaciones o heridas tratadas satisfactoriamente. Siempre se ha entendido al médico como el poseedor de un cuerpo de conocimientos, teórico y práctico, que puede ser usado para tratar al enfermo.

Pareciera que lo afirmado en el párrafo anterior fuera fácilmente materia de consenso entre aquellos que, de una u otra manera, se vinculan con la actividad médica. Desde ya, no parece muy osado afirmar que, desde la aparición del ser humano como viviente racional, debe haber surgido al menos la aprehensión de algo malo que le ocurría con cierta frecuencia. Ese algo anormal, visualizado no sólo en otro sujeto sino también padecido personalmente, era vivido o percibido en el transcurso vital de los individuos. Era un evento no esperado y no deseado por el hombre, y también temido, seguramente por constatar que, en muchos casos, su aparición se asociaba gradualmente con la muerte del individuo afectado o se reproducía rápidamente en otros sujetos de la comunidad.

Ese mal constatado en otros o experimentado personalmente ha sido conceptualizado no sin dificultad a través de la historia del hombre. La aprehensión intelectual de ese hecho pudo ser fácil en algunos casos, como en accidentes que provocaban un daño estructural evidente. Sin embargo, aun el día de hoy, existen otras situaciones que impactan significativamente a las personas y no es fácil considerarlas en el mismo género de afecciones.

¿Qué es la enfermedad? ¿Qué tipo de realidad representa?, han sido preguntas que no sólo han intrigado intelectualmente a la humanidad a través de los siglos, sino que han determinado las actitudes de los hombres hacia ella(1).

Fue Hipócrates y su escuela, precedido por Alcmeón de Crotona, quien desligó la magia y la religión de la enfermedad(2:74). Por primera vez se entendió a esta como un acontecimiento que podía responder a una causalidad y una dinámica distintas de la arbitrariedad de la acción de un poder mágico o algún dios. La manera de presentarse podía dar también luces de su causa, para evitarla o hacerla desaparecer.

Este nuevo concepto de enfermedad fue lo que permitió finalmente que se constituyera la fisiología como ciencia y la medicina como un saber práctico, con metodología propia, capaz de investigar y dar razón de su actuar y, por tanto, de ser enseñada. Se diferenció así la medicina de la filosofía $(2: 87)^{1}$, de las ciencias naturales y de la mera experiencia, esta última incapaz de dar explicación de sus acciones.

La medicina hipocrática se estableció entonces como un arte, separándose primero de la superstición y de la religión y luego también de la filosofía, constituyendo así un saber con su propio método $(3, \text { cap. 12) })^{2}$. La investigación y la enseñanza del conocimiento adquirido dieron fe de su estatuto de saber propio de un tekhnites (artista, técnico) $(4: 19)^{3}$, capaz de dar razón de su actividad; entonces es una tékhne, porque su finalidad es la obtención de un efecto guiado por un conocimiento de tipo práctico, fundado en la ciencia (episteme). No busca un conocimiento especulativo propio de la filosofía y de las ciencias particulares, pero así como todas las artes, la medicina se sirve de las demás ciencias para alcanzar su propia finalidad, de modo análogo a como la ingeniería utiliza el conocimiento de la ciencia matemática, la agronomía el de la botánica, la navegación de la astronomía o la carpintería de la geometría.

Pero no solamente necesita de algunas ciencias particulares como la biología, la anatomía o la fisiología, sino

1 “...los autores del Corpus Hippocraticum van a adoptar actitudes distintas. Tres grupos principales pueden señalarse entre ellos: 1 . Los que pretenden hacer de la filosofía fundamento expreso e ineludible del saber médico (Sobre las carnes, Sobre las ventosidades, Sobre la dieta). 2. Los que, polemizando a veces con algún filósofo en nombre del saber médico (contra Meloso, por ejemplo, en el caso de Sobre la naturaleza del hombre), no vacilan en recurrir a nociones y modos de pensar más o menos "filosóficos" (Sobre la naturaleza del hombre, Sobre los lugares en el hombre). 3. Los que tratan de hacer de la medicina un saber independiente de la filosofía; y a la cabeza de ellos, el autor de Sobre la medicina antigua".

2 "Lo que digo es que no se puede rechazar la medicina antigua como inexistente o que no ha investigado correctamente, por no ser exacta en todas sus modalidades. Más bien creo que, por lo muy cerca que pudo llegar de la verdad, partiendo de una gran ignorancia, son dignos de admiración sus descubrimientos, alcanzados por el camino correcto y no por azar".

3 La medicina, tal como se la entendió a partir de la escuela hipocrática, es la que corresponde a una tékhne, entendiendo esta como una actividad poiética, pero dirigida por la razón, ya que "la tékhne no consiste en hacer las cosas, sino en saber hacer las cosas". 
también del aporte de otros saberes que no son ciencias. Así, la experiencia que reconocemos en algunos galenos -capaces de un diagnóstico o implementación de una terapia sin que ellos mismos puedan dar las razones que los fundamenten- atestigua que, de alguna manera, ese conocimiento es arma importante. También la prudencia -entendida como aquella disposición que perfecciona al intelecto en la elección del mejor medio para alcanzar un fin propuesto- junto con las demás virtudes morales hacen que ciertos médicos sean dignos de ejemplo y de referencia para otros $(5: 121)^{4}$. Por último, y siendo el hombre el objeto material de la actividad médica y su compleja realidad, podemos decir que, además de las ciencias, del conocimiento y las virtudes morales, necesita de una perspectiva que logre la aprehensión esencial del ser humano, esto para comprender los límites y exigencias éticas en el actuar del médico. Esa aproximación es el aporte de la filosofía.

La medicina, tal como la consideraron los griegos, requiere de muchos saberes para su adecuado funcionar, pero es esencialmente un arte, una tékhne, con su método propio. Tiene una finalidad externa al agente y busca un efecto que pretende obtener dando razón de los medios que utiliza. Investiga, para obtener el conocimiento práctico que le permita mejor alcanzar el efecto que busca, y es capaz de enseñar y trasmitir conocimientos y destrezas a otros que se inician en la actividad.

El establecimiento de la medicina hipocrática como un saber práctico fundado en la ciencia, diferente de otros saberes y especificado por esta nueva concepción de enfermedad, tuvo como consecuencia, además, la constitución de un nuevo tipo de médico, un sujeto conocedor y poseedor de los conocimientos y destrezas necesarias para realizar la labor que se le encomendaba. Así, por tanto, se obligaba a sí mismo al cumplimiento de su tarea de una manera acorde con la dignidad de ella. Nace entonces la profesión médica, con todo un bagaje de obligaciones intelectuales y de carácter moral, que rige sin grandes variaciones hasta nuestros días.

4 La prudencia es una virtud intermedia entre las puramente intelectuales y las morales; se distingue de las demás virtudes intelectuales y morales y también se distingue del arte, aun perteneciendo este al entendimiento práctico, porque el fin de la prudencia es intrínseco, y el de aquél extrínseco.

\section{El objeto de la medicina}

Toda ciencia, todo arte, situándonos desde una perspectiva aristotélica, es especificado como tal por su objeto. Así, por ejemplo, el objeto de la biología son los seres vivos, mientras el objeto de la arquitectura son las edificaciones. Pero tanto los seres vivos como las edificaciones son también objetos de otras ciencias o artes: los vivientes de la zoología y las edificaciones de la ingeniería; luego, debemos distinguir el objeto material -referido al sujeto en cuanto poseedor de determinados aspectos- del objeto formal, referido sólo a alguno de esos aspectos o determinaciones(6:148-161).

Sin duda que el objeto material de la medicina es el hombre, el hombre completo, el hombre como viviente racional, el Homo sapiens, el hombre como sustancia, sujeto de cambios accidentales desde su aparición en el ser hasta su muerte o corrupción. Este hombre es también objeto material de otras ciencias o artes, como la antropología, la anatomía, la escultura, la fisiología, la política, la pedagogía etc., ahora bajo sus aspectos de cultura, estructura, estética, funcionamiento, organización social y aprendizaje, respectivamente, aspectos que van especificando las distintas ciencias y artes.

El objeto formal que especifica y distingue a la medicina de las demás artes o técnicas (tékhne) referidas al hombre es, como en todas ellas, un bien que de alguna manera lo perfecciona, ya que no tendría sentido que el ser humano desarrollara un arte o técnica que lo degrade o corrompa. Es así, por ejemplo, que el arte de la pedagogía busca perfeccionar al hombre, permitiéndole que adquiera los conocimientos necesarios para un mejor desempeńo en su ambiente cultural, o el arte de la política busca perfeccionarlo desarrollando aquellas disposiciones que compatibilicen sus intereses individuales con el bien común.

El bien buscado por la medicina es la salud, entendida como una perfección entitativa que le corresponde naturalmente al hombre, y que este por alguna razón a veces no posee, ya por haberla perdido o por no haberla poseído nunca. Se dirige entonces la medicina al hombre carente de aquel equilibrio somático o somático-psicológico que percibimos como salud, al hombre menesteroso de salud, ens indigens en el decir de Laín Entralgo(7:345), buscando -mediante la combinación del conocimiento, la observación y la experiencia clínica, más la prudencia necesaria que le confiere su sello característico, entendida así a partir 
del saber hipocrático $(3, \text { cap.3 })^{5}$ - restablecer aquel equilibrio conocido y percibido como salud.

El hombre, como objeto material, y la salud a restablecer, como su bien particular, constituyen a la medicina como actividad práctica. Pero no sólo la constituyen como tal, sino que también configuran los límites de su actividad. En efecto, el establecimiento de la medicina como un arte permite distinguir dos hechos de vital importancia para el acto médico: en primer lugar, todo arte o técnica cuyo objeto es el hombre puede - por la naturaleza de su metodología- buscar sólo el bien particular de este y difícilmente puede pretender alcanzar su bien total. En segundo lugar, los límites de su actividad están dados por la capacidad que el avance del arte le permite. Su actividad entonces se detiene cuando el método no logra alcanzar lo buscado, y también cuando, pretendiendo abarcar más allá de su salud, seguir actuando va en contra del bien total del individuo, fin último al que debieran aspirar todas las actividades humanas.

Los límites de la medicina se establecen primariamente por el objeto formal que la constituye como arte y sólo circunstancialmente por las dificultades técnicas. El primer límite es fundamental; el bien buscado por su actividad no puede contraponerse con el bien total del individuo. El médico debe poseer entonces una cierta "idea" de cuál es ese bien que finaliza al hombre, ya que sin esa noción, aun cuando sea confusa, erraría demasiadas veces insistiendo tercamente en restablecer un mero equilibrio fisiológico, en un hombre anhelante de un bien más allá de él. Justamente, lo complejo de esa idea de bien total explica el dilema ético del médico: muchas veces puede no saber si la aplicación eficaz de su arte es todo lo bueno que merece ese hombre.

La segunda limitante, referida a los medios, conocimientos y herramientas que el sanador posee en un momento determinado, y cuya insuficiencia le impide muchas veces alcanzar la salud para sus pacientes, tiene un carácter temporal. Lo limita sólo en el aquí y en el ahora, y a la vez lo estimula para seguir investigando y desarrollando su arte. Es una barrera que lo desafía

5 "Acerca de la Medicina, pues sobre esta es mi charla, voy ahora a hacer mi disertación; y en primer término voy a definir lo que considero que es la Medicina: el apartar por completo los padecimientos de los que están enfermos y mitigar los rigores de sus enfermedades, y el no tratar a los ya dominados por las enfermedades, conscientes de que en tales casos no tiene poder la Medicina”. (Sobre la medicina, Tratados hipocráticos. Madrid: Editorial Gredos; 1983). e impulsa a vencerla. Es el primer límite, en cambio, el que marca y delimita su actuar, recalcándole que no todo lo que puede hacer le corresponde, y que acepte que es nada más un médico al cual sólo corresponde la salud de su paciente, siempre y cuando no vaya en detrimento de su perfección(8).

\section{El hombre como paciente}

Hasta este momento hemos intentado un breve resumen de lo acontecido en la historia de la medicina, haciendo hincapié en cómo se estableció este saber como actividad práctica con objeto propio. Hemos tratado también de precisar sus límites y las razones de estos. Ahora es preciso reflexionar acerca de la actividad del médico que le es más propia, es decir, lo que le corresponde verdaderamente hacer.

El médico hace medicina y su quehacer consiste en realizar acciones cuyo destino es producir salud. Recordemos que el hombre, en cuanto paciente, es un sujeto que percibe un impedimento en la consecución de los fines que se ha propuesto. Es un viviente pensante que, en el transcurso de sus planes vitales libremente trazados, capta que hay algo extraño e inesperado que amenaza su curso. Este impedimento no debe entenderse como algo externo, como una dificultad que es necesario sortear, sino como algo que emerge desde la estructura misma de ese sujeto. Esa dolencia bien pudiera ser un mal o defecto corporal, pero no es en cuanto tal que se percibe como enfermedad. Si causa aquel sufrimiento que lo hace consultar, es porque lo entiende como una amenaza a sus proyectos vitales autodeterminados.

Ese carácter de amenaza a los proyectos vitales establece entonces la dolencia o enfermedad. El impedimento interno originado en el ser mismo de este sujeto -sea o no una alteración bioorgánica- lo hace padecer, porque teme que el despliegue de sus proyectos se pueda frustrar. Por ello, fuertes dolores no se padecen a veces como enfermedad, muertes próximas en pacientes de edad avanzada no causan sufrimiento y situaciones de soledad o desamparo, en cambio, se presentan y viven como dolencias físicas(9). Es el médico el que debe buscar la forma de restablecer en su paciente el equilibrio psicológico, somático y espiritual que entendemos como salud. Ya hemos visto que la actividad del galeno corresponde al terreno de lo artístico, más específicamente a aquellas actividades en las cuales lo producido 
no es un objeto artificial sino un cambio en un ser vivo, un efecto que, en el caso de la medicina, se produce en sujetos humanos. El médico provoca un cambio en su paciente y este cambio puede ser conceptualizado como una modificación anatómica o estructural -en el caso de una intervención quirúrgica-, una modificación funcional -en el caso de la aplicación de algún medicamento que actúe sobre la actividad propia de un órgano o sistema-, o una modificación conductual por efecto de la psicoterapia.

\section{El surgimiento del acto médico}

A continuación debemos analizar lo que el artista -el médico en nuestro caso- busca provocar en el paciente y cómo logra traducirlo en una o varias acciones.

El médico debe poseer, antes que todo, una cierta "idea" de lo que pretende plasmar como cambio en el sujeto que lo consulta. No es fácil de conceptualizar, pero sí podemos decir que la formación de esa idea en el intelecto del médico está relacionada con diversos aspectos o criterios.

\section{El criterio de normalidad}

En primer lugar, es menester que el médico posea una cierta concepción de lo que es "normal" en un individuo. La normalidad a la que debe hacer referencia el galeno no es fácil de establecer; ella guarda relación con criterios de frecuencia estadística, en cuanto a características físicas y funcionales de todos los individuos pertenecientes al grupo etario y racial del paciente en cuestión. Ahora, esta consideración de normalidad, aun siendo importante en la deliberación del médico antes de su decisión y que le permite compararla con los hallazgos que hace en su paciente, es sin embargo sólo parte de los elementos de juicio con los que él debe contar.

Sería por cierto completamente insuficiente la comparación de lo encontrado en el paciente con el puro criterio de normalidad. El estudio de la estructura del cuerpo humano y el funcionamiento de órganos y sistemas de la economía humana son parte importante del bagaje de conocimientos para determinar las desviaciones de esa norma; sin embargo, la existencia de variaciones cualitativas y rangos cuantitativos de normalidad impiden una consideración absoluta de este criterio. Recordemos que lo considerado como "normal" se establece sobre la base de estudios de hallazgos frecuentes en grandes poblaciones de individuos. Si consideramos -a modo de ejemplo- un solo parámetro, como podría ser la presión arterial, veremos que los límites de normalidad se establecen, por un lado, estudiando las cifras en esas grandes poblaciones, pero también por los efectos nocivos que provoca cierta cantidad de presión arterial sobre los distintos órganos del cuerpo. Es sabido que ciertos individuos sufren esos mismos daños con cifras tensionales dentro del rango de normalidad, y otros no los padecen con cifras consideradas por todos como absolutamente anormales.

Basados en este único criterio podemos, a pesar de los reparos que posee, adelantar que el médico sí busca restablecer esa normalidad estructural o funcional. El cirujano muchas veces intenta reparar un órgano afectado o el internista incrementar la función de un sistema juzgado como disfuncionante en comparación con uno considerado normal. Pero el médico no puede lograr una rectificación anatómica o funcional perfecta a su sola voluntad, y aunque pudiese hacerlo -por el avance del conocimiento y de los recursos tecnológicos- la ausencia de un patrón de normalidad absoluto -debido a esta diversidad estructural y funcional natural en la especie humana y en todos los vivientes- hace que este criterio sea sólo relativo para la consideración de lo buscado en la acción médica. El intelecto del médico deberá encontrar entonces otros parámetros en su deliberación para elegir aquella acción particular y única que logre restablecer el sano equilibrio de un paciente.

\section{El criterio de funcionalidad operativa}

Otro criterio que el médico utiliza como "idea" del efecto que pretende provocar en su paciente es una cierta concepción de funcionalidad u operatividad integral. Conocedor de lo relativo del criterio de normalidad y también consciente de las limitaciones de su arte, debe saber que, a pesar de no poder llevar a su paciente a un estado de normalidad estructural y funcional absoluta, puede sin embargo intentar conseguir que vuelva a desenvolverse satisfactoriamente como un todo.

Muchas veces -salvo quizás frente a algunas patologías, como las provocadas por agentes infecciosos, en las cuales el galeno sí cuenta con los medios para restablecer el equilibrio somático del enfermo- el médico es incapaz de revertir la situación provocada por la noxa hasta la situación previa del paciente, o la que debiera 
tener si nunca se hubiera visto afectado. Enfrentado a estas situaciones, debe tener en su intelecto algún plan de acción para ofrecer a su paciente.

El médico debe plasmar en su paciente un cambio tal que le permita superar lo que percibe como impedimento para el despliegue de sus planes vitales, aunque ese cambio no le signifique volver a ser -ni estructural ni fisiológicamente- como era o como debiera haber sido. Estos cambios pueden contemplar intervenciones tan simples en su salud como un distinto tipo de alimentación, más complejos, como la extirpación extensa de órganos o miembros o el reemplazo de ellos por otros donados o por artefactos mecánicos y protésicos.

La exéresis -o extirpación de algún órgano comprometido por un tumor canceroso- es un ejemplo de esto. El médico, ante el dolor que la tumoración provoca en el sujeto que le consulta o el sangrado de variable cuantía y ante el pleno convencimiento de que es incapaz de volver al paciente a sus parámetros de normalidad intenta, extirpando el órgano comprometido, que la persona afectada vuelva a una condición que le permita funcionar. En este caso el médico, aunque consciente de que su paciente no vuelve a su normalidad, sabe sin embargo que logra su curación.

Este criterio de funcionalidad operativa implica la existencia en el espíritu del médico de una idea de totalidad funcional que va más allá del funcionamiento propio de cada órgano, miembro o sistema del organismo humano ${ }^{6}$, y también de la normalidad cualitativa y cuantitativa establecida por los datos poblacionales. Esta idea de totalidad funcional le permite visualizar que, a pesar de ser capaz de provocar en su paciente un daño estructural significativo, objetivo y muchas veces irreversible, el cambio o efecto buscado corresponde al ámbito de la salud; por lo tanto, le corresponde realizarlo. Mas, aunque por cierto le ayuda en su objetivo,

6 No debemos olvidar que, a pesar de que el paradigma reinante como concepto de enfermedad es el de una entidad que provoca un dańo estructural o funcional de un órgano o sistema, la concepción misma de órgano o sistema no es más que una simplificación pedagógica, útil para entender cómo cada estructura hallada en la economía humana participa en el funcionamiento como un todo del organismo. Es difícil hallar una separación funcional e incluso anatómica entre, por ejemplo, el aparato circulatorio y respiratorio, y entre ellos con el sistema nervioso, excretor, endocrino y locomotor. Es entonces perfectamente posible concebir con criterios distintos sistemas separados funcionales diferentes a los actuales. Todo esto es también válido para los órganos, ya que es también difícil separar, por ejemplo, en el riñón su función excretora, circulatoria o endocrina (N. del A.). este segundo criterio sigue siendo todavía insuficiente para comprender a cabalidad la idea o concepto del cambio que el médico desea para su paciente.

\section{El criterio de tendencialidad}

Los dos criterios anteriores se refieren a cambios en un sujeto concebido como un ente dotado de una cierta estructura considerada como normal y de un cierto comportamiento considerado como posible y deseable. Sin embargo, nada dicen de su tendencialidad, nada dicen de un ser humano con un destino a alcanzar y enfrentado a hechos que le ayudarán y otros que dificultarán su camino.

El ser humano, como todo ser natural, es un ente proyectado hacia un fin, pero la interioridad que lo constituye-y determina ese fin- es de tal condición que su destino trasciende los límites o el ambiente naturalbiológico común a sus compañeros vivientes (10:18)7. En efecto, la estructura corporal y sus facultades son formadas en el tiempo para ejercer operaciones que lo posicionan en un pie y horizonte absolutamente distinguible del resto de los vivos. Solamente esto y no su composición fisicoquímica -ni siquiera su estructura genética, sorprendentemente similar a especies consideradas inferiores, según el Proyecto Genoma Humano- puede dar cuenta de realidades tales como la cultura, la medicina, la ciencia o la filosofía, y de fenómenos como el amor donativo, la compasión, la justicia, la solidaridad o la preocupación por la acción adecuada. Su conocimiento intelectual, capaz de leer lo que son las cosas abstrayéndolas de los aspectos sensibles, le permite estar abierto a todo el universo. $\mathrm{Su}$ mundo es entonces infinito, limitado sólo por su necesidad de acceder a la interioridad de lo existente a través de la sensibilidad.

7 La pregunta sobre la existencia o no de un plan en los seres vivos ha sido discutida desde los inicios de la biología, discusión que se intensifica desde los postulados de Darwin, quien niega la existencia de la finalidad en los vivientes, y otros para quienes ese plan es evidente. "No puede negarse, ciertamente, que los seres orgánicos constituyen una de tales máquinas incomprensibles para nosotros. Pero lo que podemos comprender en ellos es el funcionamiento en un momento dado; de momento en momento puede cambiar la máquina, pero en cada momento está completa, y el engranaje de un completo rodaje puede ser revelado a nuestra inteligencia. Entonces hemos descubierto su conformidad al plan.

Si intentamos investigar las variaciones que de momento en momento sufre la máquina, nos ocupamos de la tendencia hacia el fin que desde el huevo origina la gallina". (Von Uexkül J. Ideas para una concepción biológica del mundo. Madrid: CALPE; 1922). 
Ese conocimiento intelectual o racional, que distingue al ser humano del resto en la escala de los seres vivos, le permite decidir y por lo tanto ser libre. El sentido de su operación no está garantizado por una armonía automática y por lo tanto infalible, propia de los seres desprovistos de intelecto, sino que todos sus impulsos biológicos están de algún modo bajo la razón, la que debe, entendiendo el sentido de sus propias tendencias biológicas, buscar libremente el camino que le conduzca a su plenitud.

Lo que distingue al hombre es entonces lo que lo constituye como tal, y hay que comprenderlo como un ser natural con capacidad de entender lo que son las cosas en sí mismas. Es necesario entenderlo también como un viviente terrenal con impulsos y apetencias fisiológicas, necesitado de un cuerpo y de órganos sensoriales para acceder al mundo que le es propio. Mas la armonía de sus impulsos biológicos no está asegurada, como decíamos, por una coordinación automática y preestablecida, sino que regida por un conocimiento racional, que le otorga libertad para buscar y elegir las maneras o el camino que lo actualicen completamente.

Comprender así al hombre es entenderlo como un ser viviente con un destino abierto, que, al captar el sentido de cada una de sus tendencias naturales, puede autootorgarse alcanzar su plenitud donde su razón le muestre que esté. Su libertad le permite además determinar qué le conviene y qué lo degrada y corrompe.

El médico, como persona, debe entonces poseer alguna idea de lo que es el hombre y de lo que le conviene o lo perjudica para alcanzar su destino a través del despliegue de sus potencialidades, es decir, debe tener alguna idea de lo "bueno" para él. El médico es incapaz de saber qué es todo lo bueno para un paciente en particular; no puede saber cuáles son sus aspiraciones personales ni sus temores y requiere que él se los explicite. Sin embargo, no podría conceptualizar una intervención sin tener al menos suficientemente claro lo que perfecciona a los seres humanos en general.

El médico enfrenta numerosas situaciones en las cuales el paciente solicita una intervención que lo perjudicará en su estructura psicosomática. Y el médico debe hacer un esfuerzo para explicarle los alcances de su petición. Otras veces, el tratante percibe que ciertas conductas de su paciente atentan contra el despliegue de lo que le corresponde como sujeto humano. Así como el jardinero sabe que cierta cantidad de agua y de sol va a beneficiar a las plantas del jardín a su cuidado, el médico como persona también sabe, sin necesidad de mayor interacción con su paciente, que ciertos hábitos de alimentación o ciertas conductas sexuales lo exponen no sólo a un riesgo de salud, sino que atentan claramente contra lo que le corresponde como un sujeto de naturaleza personal.

¿Cómo podría el médico determinar esto sin saber lo que es un hombre y una mujer? ¿Cómo podría el médico concebir una intervención sin una consideración global e integral del sujeto humano? La consideración de la mujer y del hombre sólo como una estructura y funcionalidad fisiológica es insuficiente para "idear" una acción médica. Es preciso que, en su plan de acción el galeno no sólo conciba un efecto que acerque nuevamente a su paciente a lo que de alguna manera se considera como normal -o si eso no es posible le restablezca una funcionalidad en su desempeño-, sino que además le permita, le facilite -y en ningún caso le impida- el despliegue de su naturaleza humana.

La normalidad estructural y la funcionalidad operativa como criterios organizativos - con todas las dificultades que representan estos conceptos-, y junto con ellos esta consideración teleológica del sujeto humano, son entonces indispensables para que el intelecto del médico logre concebir un cambio que satisfaga el ansia del sujeto consultante, que se percibe como salud. Sin embargo, todavía estos tres elementos no son suficientes, porque no podemos olvidar que la naturaleza humana, aunque se materializa en representantes de esta especie, existe individuada en sujetos personales.

\section{El paciente como sujeto único y original}

Podemos afirmar que, en definitiva, todos los individuos de la especie humana tienden a un mismo fin último y que este corresponde a su felicidad plena. Sin embargo, también podemos advertir que cada ser humano busca su propio camino para alcanzarla. La misma racionalidad que lo constituye esencialmente le permite y le obliga a determinar su andar. Cada mujer y cada hombre le da sentido a su propia existencia tomando primero conciencia de lo que le fue legado -constitución física, ambiente cultural, condición de nacimiento, etc. - y estableciendo después su propio andar mediante sus libres decisiones, sorteando, aceptando o luchando con los avatares externos. Todo este 
camino constituye su biografía, existiendo una para cada individuo. La vida humana podemos entenderla como una historia en la cual cada individuo va tomando los hechos vividos como acontecimientos, en cuanto significativos para ese camino que se autotrazó(11:142-159).

Cada ser humano es entonces un universo distinto de planes, sueños, aspiraciones y metas. Cada persona es distinta en sus anhelos y preocupaciones. Cada una de ellas, además de su realidad ontológica como sujeto perteneciente a la especie humana, tiene entonces - producto de sus propias circunstancias y decisiones personalesroles y responsabilidades distintas. Cada uno percibe y se relaciona con su medio de manera diversa. Todo lo captado del mundo externo resuena en el interior de cada hombre y de cada mujer de distinta manera: un mismo paisaje o una misma mirada provocan perturbaciones internas diversas en cada persona, o un mismo hecho puede ser inadvertido para uno y puede constituir un acontecimiento importante para la vida de otro.

Este universo de realidades humanas también se presenta a la consideración de un médico. Cuando un sujeto consulta por alguna dolencia es todo este mundo diverso el que consulta. Es un individuo que no sólo tiene una determinada estructura física -que funciona adecuadamente o no-, a quien no sólo le corresponde determinar su futuro guiado por su razón, sino también es un individuo personal que es padre, madre, hermano e hijo. Tiene una actividad o profesión, tiene responsabilidades, planes, temores $\mathrm{y}$ preocupaciones; tiene experiencias, creencias, opiniones y certezas. Proviene de una determinada cultura con la cual se identifica. Estará, por tanto, dispuesto a someterse a ciertas acciones médicas y no a otras, porque tiene ya formada una opinión o certeza de lo que es bueno o malo para su proyecto de vida. $Y$ es a toda esta compleja realidad a la que el médico debe "idear" una intervención, para que reestablezca ese equilibrio que percibimos como salud.

Además, cada patología es vivida de distinta manera entre un individuo y otro, dependiendo de sus características, vivencias, creencias, situaciones, perspectivas o planes personales. Cada opción terapéutica, entonces, deberá ser considerada tomando en cuenta estos aspectos. Por esto, es frecuente que un médico decida tratar de una determinada manera un trastorno específico en un paciente y decida no hacerlo -o hacerlo de otra manera- en otro. Es que el médico, más que especialista en determinada área de la ciencia médica, debe constituirse en "especialista" de ese paciente en particular. Debe saber captar todo ese universo existente en un paciente con nombres y apellidos, $y$ luego concebir una intervención que para esa persona en particular represente su curación. Para ello será necesario que la escuche y logre el clima de confianza necesario, de modo que ella pueda explicitarle todo aquello de su especial intimidad que le sirva al tratante para restablecer su salud.

Es aquí y no en otra parte donde cobra sentido el llamado principio de respeto por la autonomía del paciente, que no debe entenderse como que el médico deba ser el ejecutante técnico de los deseos del consultante, sino como la única manera que el médico tiene para conocer a cabalidad lo que es bueno para él. En consecuencia, el médico, aunque considere los criterios de normalidad, funcionalidad y tendencialidad genéricas del ser humano, no puede saber ipso facto lo que es bueno para un paciente singular. Entenderlo como el sujeto único y original que es le permitirá proponer la intervención "ideada" específicamente para él.

\section{Recapitulación y conclusión}

Como podemos percibir en el último aspecto analizado, el médico se encuentra con una infinitud de sentimientos, aspiraciones y roles distintos, tantos como pacientes existen. Todos los criterios esbozados poseen, de alguna manera, un cierto patrón, una cierta referencia a la cual el médico puede apelar para concebir una intervención curadora. El conocimiento cierto de varias ciencias sirve de guía al intelecto del médico: la anatomía, fisiología, antropología, etc. Mas todas ellas se refieren a un hombre concebido universalmente, es decir, a todos los hombres y mujeres pero en definitiva a ninguno en particular. Esto sencillamente porque no hay ciencia humana de lo particular, o sea, no hay ciencia posible que pueda guiar al médico a "idear" una intervención que sane a un paciente particular que está revelando su intimidad única y original.

De alguna manera entonces el médico debe crear -respetando los parámetros de normalidad, funcionalidad y tendencialidad - un efecto en un individuo particular que, considerando su originalidad personal, satisfaga su aspiración de salud y se perciba con este efecto sanado. Una vez ya concebido este efecto, debe, aplicando las leyes de su oficio, plasmarlo en su paciente con prontitud y seguridad. En el acto médico existe entonces un acto 
de "creación" individual, único y personal, surgido de la subjetividad del médico y dirigido específicamente a un paciente en particular.

Es esta "creación" personal, surgida del médico y dirigida a un paciente único e irrepetible, lo que se olvida cuando se intenta conceptualizar la actividad médica como desvinculada del médico, no sólo como agente moral, sino también como agente causal. La tesis de la llamada "medicina basada en evidencias", especialmente con sus corrientes más extremas, ha penetrado lamentablemente en la teoría de la enseñanza de la medicina, con la pretensión de que la decisión del clínico es solamente el fruto de un análisis cuantificado de la experiencia recopilada en la literatura médica en grupos de pacientes -lo más parecidos posibles-al caso particular que un médico enfrenta(12).

Queda así en evidencia la dificultad que subyace en esta manera de enfrentar la decisión médica, ya que es imposible encontrar, en toda la extensión de lo publicado en la literatura médica, la respuesta a la pregunta que el médico se hizo, pues no existe ningún "buscador" electrónico que permita encontrar una respuesta para un paciente particular. No existe ni existirá jamás un trabajo metodológicamente validado que logre responderla. Naturalmente, se puede ir acotando la búsqueda a trabajos clínicos en grupos de pacientes parecidos a la realidad del paciente en cuestión, pero no podría existir un trabajo en pacientes exactamente iguales al que presenta el problema.

La razón última de la imposibilidad para encontrar en la literatura la respuesta a la pregunta que se hizo el médico radica en la originalidad e incomunicabilidad que un paciente único, con nombre y apellido, presenta como sujeto personal. El médico no puede sustraerse a ellas y, por ende, toda pregunta clínica acerca de este paciente se la hará tomando en consideración su compleja realidad, y naturalmente toda respuesta deberá contemplarla. Como no existen tipos de pacientes, toda pregunta y toda respuesta deberá ser "a la medida", creada especialmente para esa persona en particular en sus especiales circunstancias.

También hay intentos de establecer parámetros en el ámbito de la salud pública. Para disminuir o redirigir los gastos de salud y mejorar por lo tanto la administración de los recursos siempre escasos de un país, se establecen atenciones e intervenciones "tipos" para las diversas patologías. Así, por ejemplo, los intentos para que a pacientes que presentan una neumonía se les solicite los mismos exámenes de laboratorio y se les apliquen los mismos antibióticos. Esto se aplica también en Chile con la nueva ley que modificó las acciones de salud, estableciendo un número de patologías que tienen ciertas garantías explícitas exigibles de calidad, accesibilidad, oportunidad y costo(13). Las ventajas buscadas son evidentes. Cuando se trata de administrar recursos, es más fácil hacer presupuestos y contener costos conociendo de antemano el número de pacientes que se estima presentará una patología determinada, y cuánto costará el tratamiento de cada uno de ellos. El establecimiento de "paquetes" de atención busca conseguir ese objetivo administrativo.

Estos esfuerzos, aun con indiscutible valor administrativo, llevan a una insatisfacción en los pacientes respecto de la atención médica que reciben, debido a que si bien mejoran ciertos aspectos deficitarios de su atención de salud, se descuidan otros fundamentales para lo que ellos aspiran. El establecimiento de estas atenciones tipos, que encasillan a todos los pacientes en grupos, no considera lo que con ahínco hemos postulado y repetido -y que han repetido hasta el cansancio a través de generaciones los profesores de medicina-, respecto de que no hay enfermedades sino enfermos. No pueden establecerse por tanto atenciones tipos ni promedios, ya que el paciente requiere una respuesta sólo para él, en sus particulares circunstancias vitales. El único capaz de ofrecérsela es un médico que la "cree" para él y se la aplique utilizando la virtud de su arte.

También vemos, en el último tiempo, la implementación de medidas tendientes a reducir las llamadas "listas de espera" de enfermos con patologías susceptibles de soluciones quirúrgicas. Se contratan para ello a médicos que viajan especialmente a realizar actos quirúrgicos a pacientes que conocen recién en el pabellón de cirugía y a los que no verán más después de terminada la operación. Estos médicos actúan sólo como ejecutores de una técnica quirúrgica. A pesar de la corrección de lo dañado, la insatisfacción de esos sujetos será inevitable. Nada impedirá que cada uno de ellos sienta haber sido tratado como una máquina a la que se le cambió una parte descompuesta.

Esta reflexión no es fácil de ser explicitada y/o comprendida, y eso explica en parte que no se consideren estos temas al momento de establecer políticas sanitarias o administrativas. Sin embargo, podemos decir que en 
el fondo de estas políticas subyace una consideración reducida de lo que es el paciente y por lo tanto una mujer o un hombre. La naturaleza de la acción médica requiere, para su comprensión, de un adecuado conocimiento de la naturaleza humana, de lo que es un paciente y en lo que verdaderamente consiste la relación entre él y su médico. Debemos entonces abogar para que ese íntimo contacto interpersonal que constituye el acto médico no pierda su verdadero sentido; procurar que los valores de la profesión médica, que se actualizan cada vez que un paciente solicita atención, se mantengan aun en las intervenciones clínicas más simples y cotidianas. En definitiva, que para un médico el paciente siempre sea un individuo con la dignidad que le otorga su estatuto de persona.

\section{Referencias}

1. Serani A. La Enfermedad Humana. Medicina y Ética 1999; 10(3): 333-346.

2. Lain Entralgo P. Historia universal de la medicina. Barcelona: Salvat Editores S.A.; 1982.

3. Sobre la medicina, Tratados hipocráticos. Madrid: Editorial Gredos; 1983.

4. Zubiri X. Cinco lecciones de filosofía. Madrid: Alianza Editorial; 1992.

5. Aquino T. De las virtudes. Santiago de Chile: Universidad de los Andes; 1997.

6. Maritain J. Filosofía de la naturaleza. Buenos Aires: Club de Lectores; 1980.

7. Laín Entralgo P. Antropología médica. Barcelona: Salvat Editores; 1985.

8. Besio M. Sobre el acto médico. Cuadernos de Bioética 2003; 50(14): 25-38.

9. Besio M. El feto como paciente. En: Guzmán E. (editor). Selección en temas de ginecoobstetricia. Santiago de Chile: Ediciones Publimpacto; 2005: 485-492.

10. Von Uexkül J. Ideas para una concepción biológica del mundo. Madrid: CALPE; 1922.

11. Peña Vial J. La poética del tiempo, ética y estética de la narración. Santiago de Chile: Editorial Universitaria; 2002.

12. Clancy C, Cronin K. Evidence-Based Decision Making: Global evidence, Local decisions. Health Affairs 2005; 24(1): 151-162.

13. Ley 19.966 de Régimen General de Garantías en Salud. República de Chile.

Recibido: 16 de enero de 2009

Aceptado: 18 de marzo de 2009 